\title{
Modelo dinámico ecológico de desarrollo humano de la deserción escolar en Aymaraes, Apurímac
}

Dynamic ecological model of human development of the school desertion in Aymares Apurimac ${ }^{1}$.

Grace EsPINOZA², LOURDES CARPIO ${ }^{3}$

Universidad Nacional Mayor de San Marcos, Lima, Perú

(RECIBIDO 02/01/2015, ACEPTADO 10/06/2015)

\section{RESUMEN}

El presente artículo informa un estudio cualitativo sobre la deserción escolar en Aymaraes, Apurímac. El objetivo del estudio fue caracterizar los factores involucrados en la deserción escolar, mediante un Modelo Ecológico de Desarrollo Humano de Urie Bronfenbrenner. Los diferentes factores reportados como causas de la deserción escolar se organizan de acuerdo al tipo de entorno que constituye para el estudiante que deserta.

Macrosistema, Exosistema, Mesosistema y Microsistema. Este último sistema organiza los aspectos psicológicos del estudiante, y es explicado a través de la teoría de desarrollo personal de Pedro Ortiz. Los resultados dan cuenta que de la interacción positiva o negativa de cada uno de los factores involucrados en la problemática, por sí mismos no determinan el problema. La interacción de cada uno de estos factores se analizan mediante la dinámica de sistemas, de cada uno de los factores del sistema y su interacción es que emerge la deserción escolar.

Palabras clave: Deserción, escolar, teoría de sistemas, dinámica de sistemas, modelo ecológico de desarrollo humano, sistemas de personalidad.

\footnotetext{
ABSTRACT

The present article reports of a qualitative study in the school desertion in Aymaraes Apurimac. The aim of the study was to characterize the factors involved in the school desertion, by means of an ecological model of human development of Urie Bronfenbrenner.

The different factors brought as reasons of the school desertion, are organized, in agreement type of environment that they constitute for the student who deserts. Macrosystem, exosystem, mesosystem and microsystem. The latter system organizes, the psychological aspects

1 La investigación fue realizada en los años 2008 y 2009, financiando con el Fondo de Promoción de Trabajo de Tesis: Facultad de Psicología. Análisis dinámico la deserción escolar en la provincial de Aymares: una propuesta de estudio desde un enfoque ecológico del desarrollo humano.

2 Tesista. E-mail: grace.pardo@gmail.com

3 Asesora de la investigación, Docente asociado de la Facultad de Psicología UNMSM. E-mail: lucemarina@hotmail.com
} 
of the students, and is explained across the theory of the Personal development of Pedro Ortiz. The results realize of the positive or negative interaction, of each of the factors that involved in the problematics for themselves, do not determine the problem: the interaction of every each one of these factors is analized by means of the dynamics of the systems. Of each of the factors of a system is that it emerges the school desertion.

Kay words: Scholar desertion, teory of the systems, dynamics systems, ecological model of human development, personality systems.

\section{INTRODUCCIÓN}

La provincia de Aymaraes está ubicada al sur este del Perú, es la provincia con mayor extensión territorial de Apurímac. Está divida en 17 distritos, su capital es el distrito de Chalhuanca (Plan de Desarrollo Concertado Aymaraes, 2006). La población urbana es de 12,764 personas, mientras que la población rural es de 16,805 (INEI, 2007).

La cantidad de personas que viven en pobreza representa el 70,8\%, del total de la población. Del total de la población de Apurímac, Aymaraes representa la quinta provincia con más habitantes pobres Su población continuamente emigra a ciudades de Lima, Cusco, Arequipa, Ica y Ayacucho (INEI, 2007) Tienen una de las tasas de mortalidad infantil más altas de la región $(27,2 \%)$. Los problemas sociales que afectan a la población aymarina son, además de la pobreza extrema, el nivel de analfabetismo con respecto a la región (17\% en las zonas urbana y el $27,7 \%$ en las zonas rurales). El idioma más hablado por la población es el quechua $(64,8 \%)$ (INEI; 2007). A esto se le suma uno de los problemas sociales que afectan el sistema educativo: la deserción escolar.

Para estudiar la deserción escolar consideramos esta problemática como un fenómeno social, que no tiene una sola causa o determinante, sino como un fenómeno que emerge de la interacción de todos los posibles factores que influyen en el alumno que deserta. Por lo que conviene ser tratado y estudiado como un sistema social. Por ello se investiga la deserción escolar en la provincia de Aymaraes, Apurímac, desde una perspectiva cualitativa que permita comprender el fenómeno a través de un análisis dinámico de la interacción de los factores involucrados, los cuales se encuentran organizados en un modelo ecológico del desarrollo humano (Bronfenbrenner, 1979 ), para comprender cómo los ambientes en los que transitan las personas cumpliendo roles determinados, tienen impacto sobre las fuerzas que afectan directamente su desarrollo psicológico. En el centro mismo de este modelo, se representan la configuración de los fenómenos psicológicos del alumno que deserta, organizados en los sistemas afectivo-emotivo, cognitivo-productivo y conativo-volitivo de la personalidad (Ortiz, 1994).

\section{La deserción escolar}

La deserción escolar es uno de los problemas que actualmente enfrenta la educación en el mundo entero, principalmente en aquellas regiones donde lo índices 
de desarrollo humano están por debajo del nivel promedio (PNUD, 2007). En Latinoamérica la deserción escolar es uno de los principales desafíos (Espíndola y León, 2002; CEPAL; 2002; PREAL, 2003).

El Perú no podría estar ajeno a esta problemática, donde se sufre las tasas más altas de repetición y abandono escolar, donde 3 de cada 10 jóvenes en edad de acceder a la educación secundaria no lo hace, y en las zonas rurales aproximadamente 5 de cada 10 jóvenes se encuentra en esta situación (Alcázar, 2008). Según la Encuesta Nacional de Hogares el 13,0\% de la población entre los 3 y 16 años de edad no asiste al colegio (INEI, 2007). Si se hace una comparación del índice de deserción escolar por niveles de enseñanza, se tiene que la educación inicial muestra mayor índices $(36,0 \%)$, seguida por la educación secundaria $(29,9 \%)$ y por la educación primaria (5,7\%) (Ministerio de Educación, 2005).

Asimismo, diversos estudios nos indican que la proporción de desertores se incrementa con la edad de manera significativa, y que es mayor en la sierra y selva peruana, así como también es mayor para las mujeres, principalmente en las zonas rurales (Cueto, 2004; Alcázar, 2008). Así vemos que en Apurímac, una región eminentemente rural, los niños que culminan la educación primaria oportunamente son el $63 \%$, mientras que los jóvenes que culminan secundaria oportunamente son el 35, 3\%. Por otro lado, la población joven con secundaria completa es de 55,3\%, y la tasa de analfabetismo en la regiones de 23, 1\%(Ministerio de Educación, 2005). Entre tanto para la población joven, solo el $47 \%$ de jóvenes de entre 20 y 24 años tiene secundaria completa (INEI, 2007).

En la provincia de Aymaraes, los niños que culminan la primaria oportunamente solo son el $69,6 \%$, y los jóvenes que culminan la secundaria oportunamente son el $34,3 \%$. Mientras tanto, la población joven que cuenta con secundaria completa es de 58,1\%(INEI, 2007). El gráfico N ${ }^{\circ} 1$ muestra una comparación del total de alumnos retirados durante los años 2007, 2008 y 2009 en la provincia de Aymaraes por niveles educativos. El informe anual de la UGEL Aymaraes (2007), indica que la deserción escolar en el nivel inicial es del 8\%]; en la educación primaria es del $6.7 \%$, y en el nivel secundario es el 10, 36\%).

Gráfico 1.

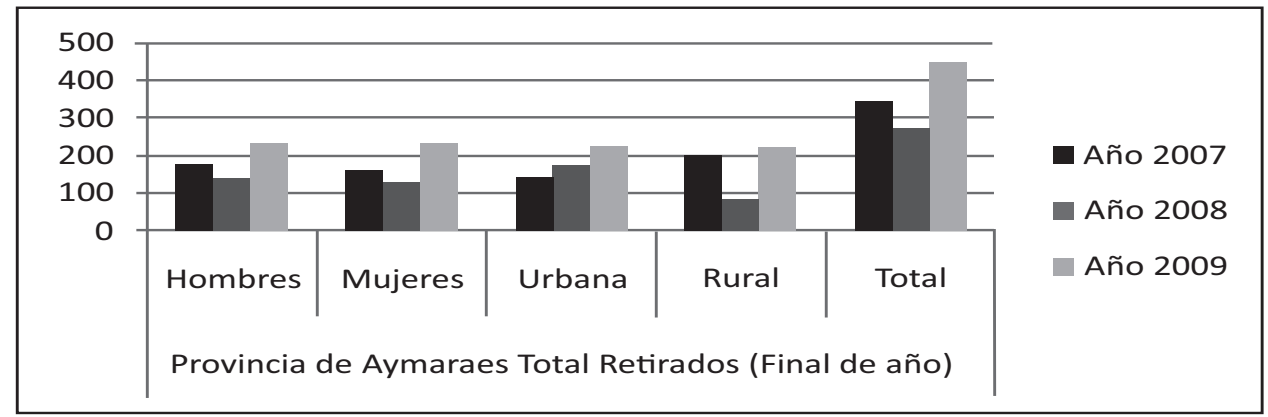

Fuente: Informe consolidado 2007. UGEL Aymaraes, INEI y ENAHO. 
La mayoría de investigaciones sobre deserción escolar en el Perú, busca dar cuenta de variables causales de la problemática. Importantes estudios en base a aplicación de encuestas a estudiantes regulares y jóvenes desertores, indican que los factores que contribuyen a la explicación de la conducta de los jóvenes en torno a la deserción son de tipo individuales, familiares, referidos a la escuela y a la comunidad (Cueto, 2004; Alcázar, 2008).

Entretanto, existen indicadores diferenciales entre los desertores de zonas urbanas y rurales. En las zonas rurales, las principales razones que permiten la deserción serían la necesidad de trabajar, el embarazo adolescente (Lavado y Gallegos, 2005).

Asimismo, los estudios indican factores externos asociados como por ejemplo, que la época de mayor deserción escolar se da después de las vacaciones de medio año, atribuido a la demanda de mano obra para los trabajos agrícolas (Cueto, 2004).

En base al resultado de este tipo de investigación, en su mayoría cuantitativas, se han ido implementando estrategias que permitan aminorar la manifestación del problema a partir de la consideración de cada factor estudiado hasta el momento. Es así como se ha organizado y replanteado los objetivos de la educación enmarcados en el Plan Nacional, el cual incluye la implementación y ejecución de programas para mejorar la calidad educativa, para lo cual capacita a docentes, mejora infraestructuras, crea condiciones para incrementar la cobertura educativa y propone alianzas para crear condiciones sociales que permitan al estudiante lograr su formación educativa básica (salud, vivienda, ingreso económico, etc.).

La estrategia implementada para superar las problemáticas educativas, incluyendo la deserción escolar, parten de una información parcial del problema, es decir se sigue una ruta lógica de causa efecto, más no parten de un análisis que permita identificar de qué manera dichos factores interaccionan, para a partir de ello comprender el fenómeno a cabalidad. No se tiene información del alcance o nivel de influencia de cada factor encontrado sobre la variable principal.

\section{Modelo utilizados para organizar la información y el análisis}

\section{Modelo Ecológico de Desarrollo Humano}

El modelo ecológico de desarrollo humano fue propuesto por Urie Bronfenbrenner, para estudios en el ámbito de la psicología del desarrollo. Esta teoría comprende el estudio científico de la progresiva acomodación mutua entre un ser activo, en desarrollo, y las propiedades ambientales de los entornos inmediatos en los que vive la persona en desarrollo, en cuanto este proceso se ve afectado por las relaciones que se establecen entre estos entornos, y por los contextos más grandes en los que están incluidos los entornos.

El desarrollo es definido como la concepción cambiante que tiene una persona del ambiente ecológico y de cómo se relaciona con él, así como su capacidad para descubrir, mantener o modificar las propiedades del ambiente en que se encuentra (Bronfenbrenner, 1979). 
El paso de una persona de un ambiente a otro implica cambiar de rol o de entorno siempre, esto se denomina transiciones ecológicas. Esto es una clara representación de los continuos procesos de acomodación mutua entre el organismo y su entorno (Bronfenbrenner, 1979). Así, asumir un nuevo rol implica nuevas expectativas de conducta asociadas a determinadas posiciones en la sociedad (p.e. Ser hijo, ser hermano, ser estudiante, ser padre, ser madre, ser trabajador, etc.)

Bronfenbrenner define el ambiente ecológico como una disposición seriada de estructuras concéntricas, en la que cada una está contenida en la siguiente. Cada estructura concéntrica es la extensión más allá de la situación inmediata que afecta directamente a la persona en desarrollo.

Microsistema.- Es un patrón de actividades, roles y relaciones interpersonales que la persona en desarrollo experimenta en un entorno inmediato determinado, con características físicas y materiales particulares. El microsistema va a constituir el ambiente más cercano al alumno que deserta. Su hogar, el colegio, la comunidad, el ambiente de reunión con los amigos. El rol que desempeña este alumno va a depender de la configuración de los componentes de su personalidad lo cual va organizar su interacción con los demás en este ambiente.

Mesosistema.- Comprende las interrelaciones de dos o más entornos en los que la persona en desarrollo participa activamente (para un niño, las relaciones entre el hogar, la escuela y el grupo de pares del barrio; para el adulto, entre la familia, el trabajo y la vida social). Es un sistema de mesosistemas, el que se forma o se amplía cuando la persona en desarrollo entra en un nuevo entorno. Las formas pueden ser que las personas participen activamente en ambos entornos o pueden pasar de un lado a otro (Bronfenbrenner, 1979).

Exosistema.- Se refiere a uno o más entornos que no incluyen a la persona en desarrollo como participante activo, pero en los cuales de producen hechos que afectan a lo que ocurre en el entorno que comprende a la persona en desarrollo, o que se ven afectados por lo que ocurre en ése entorno (Bronfenbrenner, 1979).

Macrosistema.- Este sistema hace referencia a las correspondencias, en forma y contenido, de los sistemas de menor orden (micro, macro y exo), que existen o podrían existir, al nivel de la subcultura o la cultura en su totalidad, junto con cualquier sistema de creencias o ideología que sustente estas correspondencias (Bronfenbrenner, 1979).

\section{Sistemas y Dinámica de Sistemas}

Un sistema es un conjunto de elementos en interacción, que son el resultado de realimentaciones entre sus elementos. Estas influencias mutuas determinarán cambios en sus elementos. Por lo tanto, los cambios que se producen en el sistema son reflejos, en alguna medida, de las interacciones que tienen en su interior, y estos cambios se manifiestan mediante un comportamiento que podemos observar e intentar medir (Forrester, 1999; Forrester, 2002). 
La deserción escolar también es un fenómeno social, se produce dentro de un sistema con elementos que interaccionan entre sí, constituyendo la organización y dinámica para este tipo de sistema. Un método muy usado para estudiar las interacciones de los elementos que conforman la dinámica de un sistema es la Dinámica de Sistemas.

La Dinámica de Sistemas.- es una técnica de simulación continua que permite establecer relaciones entre las variables del sistema en estudio para lograr una visión sistémica de los procesos, lo cual lleva a identificar algunos posibles factores que crean la complejidad del sistema (Aracil, 1995).

Los diagramas causales, permiten asumir la estructura de un sistema dinámico. Esta estructura viene dada por la especificación de las variables que aparecen en el mismo, y por el establecimiento de la existencia o no existencia de una relación entre cada par de variable estudiada. La metodología también incluye el diseño de Bucles de realimentación que pueden ser positivos o negativos. Los positivos son aquellos en los que la variable de un elemento se transmite a lo largo del bucle de manera que se refuerza la variación inicial. Los negativos son aquellos en los que una variable en un elemento se transmite a lo largo del bucle de realimentación lo que determina su variación de signo contrario en el mismo elemento (Aracil, 1995).

\section{Modelo de Desarrollo de la Personalidad}

Los componentes de la personalidad del alumno se describen de acuerdo a la teoría del desarrollo de la personalidad de Pedro Ortiz Cabanillas. Estos componentes son el sistema afectivo-emotivo, cognitivo-productivo y conativo-volitivo que van a organizar el sistema de la personalidad. (Ortiz, 1994).

Sistema Afectivo-emotivo. - El temperamento se organiza desde el componente afectivo-emotivo de la consciencia. La actividad de este sistema tiene que ver con el mantenimiento de la vida del individuo y de la sociedad humana. Estructuralmente serían disposiciones afectivas (sensaciones afectivas y sentimientos), estados de ánimo y de humor, (se expresan como emociones y gestos.)Sensaciones básicas (hambre, sed, sexo, etc.); sentimientos intrapersonales (alegría, tristeza, fracaso, frustración, optimismo, depresión, etc.), interpersonales (amor, cariño, ternura, placer, deseo sexual, cólera, agravio, disgusto) y extrapersonales (sorpresa, admiración, angustia, ansiedad, temor), (Ortiz, 1994).

Sistema Cognitivo-Productivo. - En base a este sistema se organizaría el intelecto, un componente que va a organizar la personalidad, a nivel consciente, de la persona requiere de una clase de actividad cognitiva por medio de la cual el alumno pueda representar su ambiente, y desplazarse y operar en él (Ortiz, 1994). Las aptitudes que tiene la persona (conocimientos, destrezas y habilidades), sus niveles perceptivos, y atencionales, conformarían la estructura de este sistema; del intelecto del alumno, cuya actividad externa se relaciona con la actividad social productiva que se expresa por medio de acciones que constituyen el desempeño personal (Ortiz, 1994). 
Sistema Conativo-Volitivo.- Organiza la forma de accionar de las personas, integrando el comportamiento emotivo y el desempeño productivo en base a reglas morales que reflejan la estructura ética de la sociedad. Este sistema organiza el carácter de las personas, configurando los motivos y los valores* de la persona, a través de sus convicciones, expectativas, intereses, aspiraciones, intenciones, ideales, perspectivas, prejuicios, responsabilidades, obligaciones, deberes, entre otros (Ortiz, 1994).

\section{MÉTODO}

El estudio de la deserción escolar en Aymaraes se abordó desde una metodología cualitativa.

Las estrategias utilizadas para recoger información fueron: observación participante, entrevistas, talleres de diagnóstico, análisis de contenido de fuentes documentales (datos estadísticos).

Los datos de tipo descriptivos fueron recogidos a través de entrevistas a los principales actores de la provincia. Como sujetos participantes se consideraron: Docentes en ejercicio de diferentes niveles educativos de la provincia de Aymaraes; Funcionarios Públicos (UGEL, Municipalidad, Posta Medica, Juzgados y Fiscalía Mixta de Aymaraes); Representantes de las Comunidades; Estudiantes de nivel secundario en actividad y desertores; Padres de familia. Las preguntas planteaban interrogantes como preguntas como: ¿En su distrito hay casos de estudiantes que abandonan el colegio? / ¿Usted cree que esto es un problema para su comunidad? ¿Por qué? / ¿Por qué razones cree usted que los estudiantes abandonan el colegio? I ¿Qué cree usted que se pueda hacer para superar este problema?

Los talleres organizados el 2006 para discutir el presupuesto participativo del año 2009 y el Plan de Desarrollo Concertado de la Provincia de Aymaraes 2010-2021 y el Gobierno Regional de Apurímac 2010-2021 permitieron conocer las opiniones que tenían los actores sociales organizados, sobre las problemáticas de su comunidad, distrito, provincia y región. Estos talleres concluían con plenarias en las que se exponían los análisis realizados y las posibles soluciones.

Para el análisis de contenido se utilizaron como fuentes documentales: Reportes anuales consolidados 2007, 2008, 2009 de la UGEL Aymaraes; investigaciones sobre la situación nacional e internacional de la deserción escolar, y sus causas vislumbradas a través de investigaciones cuantitativas de proyección estadística; reportes estadístico anuales del INEI sobre los factores sociales asociados al problema de estudio.

\section{RESULTADOS}

Estructuración del Modelo Ecológico de Desarrollo Humano.- Esta información sumada a las propias observaciones de la dinámica de los actores sociales durante 
los años 2008 y 2009, principalmente educativos, nos permitió caracterizar el problema a través de sus variables causales. Las revisiones de investigaciones sobre la deserción escolar a nivel nacional, y principalmente en áreas rurales, nos permitió definir las variables que influirían, en la deserción escolar.

Cada una de estas variables se ha organizado y ubicado, de acuerdo a su nivel de alcance o influencia en una estructura ecológica de desarrollo humano, modelo de Urie Bronfenbrenner. (Ver Gráfico 2)

Gráfico 2. Modelo ecológico de desarrollo humano.

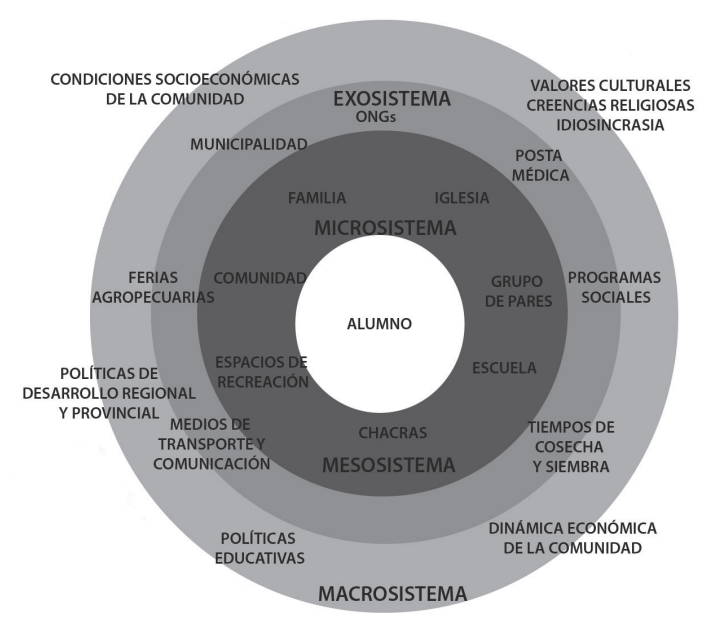

Mediante la utilización de la dinámica de sistemas se construirán los diagramas causales de todas las variables para la deserción escolar, asimismo se ubicarán los sistemas de realimentación que puedan producir los comportamientos observados. Esto permitirá realizar el análisis dinámico de estos factores en interacción.

Microsistema.- Este sistema de actividades, roles y relaciones interpersonales, cara a cara, del alumno que deserta, va a reflejarse en sus sensaciones, percepciones, motivaciones, emociones, afectos y cogniciones; procesos psicológicos que se van configurando en esta interacción permanente con su entorno más inmediato y estructuran la personalidad del alumno. Estos procesos psicológicos dan cuenta de cómo el alumno organiza determinada información del ambiente y es capaz de actuar asumiendo un rol sobre este.

Los componentes de la personalidad del alumno se describen de acuerdo a la Teoría del desarrollo de la personalidad de Pedro Ortiz Cabanillas. Estos componentes son los sistemas afectivo-emotivos, cognitivo-productivo y conativo-volitivos (Ortiz, 1994) (ver el Gráfico 3). 
Gráfico 3. Microsistema organizado en los tres sistemas de la personalidad.

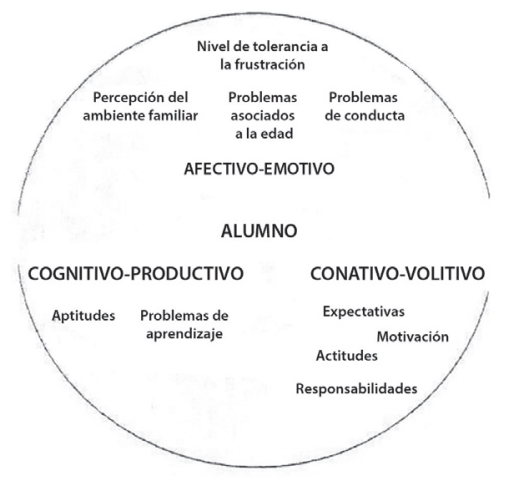

Mediante la utilización de la dinámica de sistemas se construirán los diagramas causales de todas las variables para la deserción escolar, asimismo se ubicarán los sistemas de realimentación que puedan producir comportamientos observados.

La buena configuración del sistema Afectivo-Emotivo del alumno va a depender de sus disposiciones afectivas (de rechazo o aceptación), de su estado de ánimo, de su nivel de tolerancia a la frustración, de sus sentimientos interpersonales y extrapersonales, con los que enfrente y se exprese ante las situaciones de su entorno más inmediato (v.g. sus aprendizajes, la escuela, los maestros, sus pares, su familia y la comunidad).

Entre tanto una adecuada organización del sistema Cognitivo-Productivo dependerá de los conocimientos, habilidades y destrezas del alumno, así como de su capacidad de rendimiento en las actividades escolares.

Al mismo tiempo, el sistema Conativo-Volitivo va a organizar las expectativas de los alumnos sobe la continuidad de sus estudios superiores, el nivel de sus motivaciones de continuar estudiando o elegir otra actividad, sus intereses y actitudes hacia lo que aprenden y su aprovechamiento, la decisión de desertar para asumir otras responsabilidades, y los valores que guían estas decisiones.

\section{Mesosistema}

Para nuestro modelo, en base a los datos obtenidos de nuestra entrevista, las observaciones participantes, los talleres y revisión de investigaciones previas, el mesosistema está conformado por entornos como la familia, la escuela, la actividad agropecuaria, el grupo de pares y los espacios de recreación:

- Familia.- Este es el entorno de mayor interacción para una persona. Las personas entrevistadas consideraron que la familia es el principal soporte para un estudiante; y tiene una influencia muy fuerte en la decisión que aquel tenga de desertar del colegio. Las investigaciones previas indican también que el nivel educativo de los padres influye en la preferencia y valoración de la continuidad de la educación de sus hijos,padres con mayor nivel educativo 
influirán positivamente en la percepción de sus hijos de recibir apoyo familiar para continuar sus estudios (Emerson y Pórtela,2002 citado por Alcázar, 2008). La condición de pobreza de las familias y la presencia solo de uno de los padres en el hogar, también afectaría en la decisión de desertar el colegio (Alcázar y Valdivia, 2005).De las entrevistas a los padres de familia de las zonas rurales, se puede notar una actitud no favorable hacia la educación de sus hijos. Estos padres con dedicación exclusiva a las actividades agrícolas y ganaderas, ven como sus hijos se van alejando de sus prácticas cotidianas y "olvidando los saberes" propios de la actividad en el campo, a la vez que tampoco tienen un buen rendimiento académico en el colegio, "se hacen otros (...) ni aprenden bien el colegio ni quieren ayudar en la chacra". El escaso compromiso de los padres de familia en la educación de sus hijos es remarcada por la misma comunidad, "por el trabajo también no vamos al colegio para preguntar al profesor para ver sus notas de nuestros hijos, paramos trabajando nomás, no hay tiempo tampoco" (Minkay, 2009).

- Escuela.- La escuela, el colegio, es otro ambiente de mayor integración para el estudiante. La provincia de Aymares cuenta con 29 instituciones de nivel secundario y 2 instituciones de nivel superior técnico y pedagógico. Los colegios están ubicados en la capital de los distritos o en una zona de mayor densidad poblacional; la mayoría de estos distritos son rurales. La infraestructura de estos colegios refleja la pobreza de los pueblos a que sirven, sin que el Estado busque crear un espacio de mayor atención que compense de alguna forma la situación de pobreza de la zona. La educación que se imparte en estos colegios está orientada a formar estudiantes que aspiran a una vida en zonas urbanas y costeñas, hispanoparlantes y de clase media (Ames, 2008). Las entrevistas a los padres de familia y autoridades del lugar dan cuenta de la valoración de la enseñanza a sus hijos en los colegios, "ahora ya no saben hacer ni una carta, ya no es como antes (...) ojalá les enseñaran cosas más útiles". Esta disociación entre las necesidades de conocimiento y formación de sus hijos que demanda la comunidad y la práctica real de la enseñanza con sus objetivos ajenos a esta demanda, la consideramos como indicador que favorecería la deserción.

Las prácticas pedagógicas cumplen un rol muy importante dentro del microsistema escuela. La valoración de calidad de la enseñanza de un colegio, principalmente, va a depender de las posibilidades pedagógicas que un profesor tenga en el área que enseña, de sus expectativas sobre la formación del alumno, de su satisfacción respecto a las condiciones en la que enseña y de los resultados que obtenga con sus estudiantes. La práctica de castigos físicos a los estudiantes, la enseñanza de la lectura y escritura en castellano para una población quechua hablante. Todo ello marcaría una distancia social y cultural entre el profesor y sus alumnos, así como la percepción de las escasas posibilidades de desarrollo futuro que tienen sus alumnos que incrementan las bajas expectativas de los profesores hacia sus alumnos, lo cual abriría una brecha de distancia social y cultural entre estos (Ames, 2008). 
La entrevista a profesores y pobladores de los distritos más alejados al eje vial principal de la Provincia, evidencia claramente la situación descrita. Los profesores se sienten olvidados en aquellos lugares donde no hay movilidad sino solo los fines de semana, y donde se quedan "los cinco días de la semana abandonados (...) no hay nada que hacer después de la clase, los alumnos también se van al campo, a cuidar sus animales o a sus chacras. A diferencia de los demás colegas en la capital o lugares más cercanos que tienen a su familia, tienen tele, internet, pueden estudiar también... hay todo. Acá me puedo morir también si me enfermo, no hay nada".

Los lugares alejados donde trabajan los profesores de áreas rurales va a limitar su continúa preparación, así como va a mermar sus oportunidades de capacitación (Montero et al., 2004, Ames y Vacelli, 2008, citados por Ames, 2008). Esto generaría, a su vez, una baja expectativa de sus estudiantes, limitando sus oportunidades de aprendizaje a niveles más básicos (Cueto et al., 2004; Cueto et al., 2006). La entrevista a un estudiante activo de la zona rural gráfica esta situación, "cuando le preguntamos al profesor por algún tema que no ha dicho todavía, nos dice para qué quieren saber más si solo van a estudiar en el tecnológico de Chalhuanca (...)". Las bajas expectativas y una pobre valoración del profesor sobre el desempeño y las capacidades del alumno, puede ser un indicador que también facilite la deserción.

- Actividad Agropecuaria. - La agricultura es la principal actividad económica de los habitantes de la provincia. La mayor parte de las familias se dedican a la agricultura y la ganadería la mayor parte del año, actividad en la que necesariamente participan los jóvenes en edad escolar. Aun cuando el rendimiento productivo de esta actividad es baja, generalmente por factores relacionados a las características de los suelos, el sembrío en laderas y producción bajo secano y escaso «conocimiento y manejo de técnicas agrícolas, es la principal fuente de subsistencia de la población. La alternancia de estas actividades con las del colegio, generan disminución en el tiempo de dedicación en una u otra actividad. En poblaciones rurales esta situación puede facilitar la decisión de que un estudiante deserte el colegio para dedicarse exclusivamente a la actividad agrícola o ganadera, ya que esto genera beneficios de subsistencia para su familia.

- Grupo de Pares. - Para las zonas urbanas, considerar el grupo de pares es importante. Los estudiantes que forman parte de grupos sobresalientes en lo académico, deportivo o artístico, aun cuando el ambiente familiar o la escuela influya negativamente, tendrían un ambiente solido que permitiría su mantenimiento en el colegio. La entrevista a los profesores indica "yo he podido ver que cuando algún alumno está bajo en sus notas, si él tiene el apoyo de sus compañeros y sus profesores le apoyan, por más que le vaya mal en un año, él se repone y vuelve a estudiar al siguiente año, no se va fácilmente. Los que se rinde son lo que no tienen el apoyo de sus padres y no tienen buenos amigos, ellos terminan desertando por más que el profesor le apoye". 
- Espacios de recreación.- Para los estudiantes de zonas urbanas los espacios de recreación existentes dificultan la organización del tiempo de dedicación a las actividades académicas. Las cabinas de internet, los juegos electrónicos en red y el pinball, y las losas deportivas son los únicos espacios de recreación existentes. En las zonas rurales los espacios de recreación son las losas deportivas o el campo abierto. En los talleres de los presupuestos participativos de los distritos rurales, los padres de familia y las autoridades indicaban que hacían falta espacios de recreación para sus hijos (Minkay, 2009).

Exosistema. - Para el contexto en estudio, este ambiente lo conformarían los factores indicados en el Gráfico 5.

Gráfico 5. Organización de factores que conforman el exosistema.

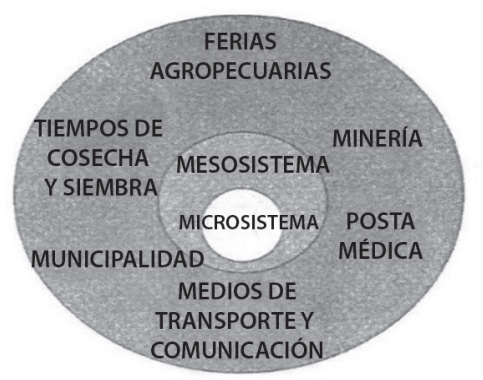

- Ferias Agropecuarias.- Se organizan dos veces al año, a finales del mes de julio e inicios de agosto, durante las fiestas patronales, en la capital de la provincia, Chalhuanca. En esta actividad participan como asociaciones $\mathrm{o}$ independientes, pequeños productores agrícolas y pecuarios de toda la provincia; la feria es muy concurrida, ya que además de la fiesta patronal, se promueve premiaciones a la calidad de los productos. En el resto del año las municipalidades, instituciones de agricultura y las asociaciones de productores, auspiciados por organizaciones del estado o empresas mineras, organizan pequeñas ferias por temporadas; estas son gastronómicas (trucha, cuy, alpaca), textiles, lácteos, miel de abeja, papa y la chirimoya. Solo en la capital de la provincia, se presentan ferias sabatinas cada quince días. Generalmente se comercian productos de la estación.

- Tiempos de Cosecha y Siembra. - La siembra y cosecha de diferentes productos demandan gran cantidad de mano de obra, se dan en temporadas específicas del año. La temporada más grande es la siembra del maíz (saratarpuy), entre los meses de setiembre y octubre. La siembra de papa es en octubre y noviembre; (papa tarpuy). La temporada de cosecha también demanda mano de obra, principalmente a los miembros de la familia. La papa se cosecha en los meses de abril y mayo, se cosecha el maíz en los meses 
de junio y julio (saratipiy). Durante estas temporadas los maestros reportan una baja asistencia de sus estudiantes, algunos de los cuales ya no retornan al colegio.

- Comunidad.- Es el espacio donde los niños y los adolescentes aprenden los modelos de comportamiento social de los adultos, la configuración del carácter de su sistema de personalidad. En la medida en que la comunidad establezca formas de valoración de la educación de sus niños y jóvenes, ya sea positiva, negativa o indiferente, influirá decisivamente en la percepción del modo de vida, y de la educación, positiva o negativa, que tengan los estudiantes y sus padres. La violencia familiar y el alcoholismo son dos problemas que afectan a los pueblos de Aymaraes. En todos los talleres y entrevistas realizadas los participantes, sin excepción, lo mencionan como un problema social de Aymaraes (Minkay zonales, 2009). Otro aspecto indicado es la debilidad en la organización comunal para enfrentar los problemas más urgentes que tiene el pueblo (Minkay, 2009).

- Gobierno Local.- Los gobiernos locales son entornos de participación inmediata de los pobladores. Es el espacio donde se deben implementar los proyectos de desarrollo para los distritos y sus comunidades, en base al análisis de sus necesidades más inmediatas y la discusión de la viabilidad de los proyectos para resolverlos. Aymaraes cuenta con un gobierno provincial y 16 gobiernos distritales. Esos gobiernos administran el presupuesto público para la población. Son encargados de construir escuela, colegio o mejorar la infraestructura existente; de construir infraestructura y obras públicas escuelas, carreteras, caminos; de promover el desarrollo social y económico local. También, de normar sobre diferentes asuntos que conciernen al bienestar, protección y desarrollo de la comunidad. En Aymaraes, los gobiernos locales asociados con el PRONAA han implementado comedores estudiantiles administrados por los clubes de madres. El pueblo señala el deficiente presupuesto, incapaz de responder a todas las demandas de la población y si acaso quieren resolver problemas sociales, las exigencias administrativas del gobierno central superan la capacidad de planificación y preparación de este tipo de proyectos (Minkay, 2009).

- Medios de Transporte y Comunicación. - Todas las capitales de los distritos cuentan con accesibilidad vial. Los hogares de las zonas urbanas cuentan con televisor, radio. La mayor parte de territorio provincial aún no cuenta con señal para telefonía móvil. Los centros educativos, a nivel provincial, están ubicados dentro de la red vial provincial, existen vías rehabilitadas y en mantenimiento que permiten el acceso rápido y adecuado a estos centros educativos, pero también hay trochas carrozables y en muchos casos solo caminos de herradura que limitan y dificultan el desplazamiento de los estudiantes de los centros poblados y comunidades alejadas. Llegar de sus casas al centro educativo les demanda hasta dos horas de caminata.

Esta situación limita un adecuado rendimiento escolar. Las entrevistas realizadas 
a estudiantes en actividad, indican "tenemos que caminar desde las 6 de la mañana para llegar 15 para las 8 al colegio (...), tenemos que levantarnos a las 4 de la mañana para prepararnos el desayuno y luego venir para el colegio; a veces si caminamos toda la carretera puede que algún carro pase y nos lleve, pero no es seguro, por eso por este lado del camino nomás bajamos". Por su parte, los profesores indican que "los muchachos llegan cansados y a veces se cansan o se duermen rápido en la clase (...), así es difícil esperar que aprendan bien lo que se les enseña, están mal nutridos".

- Minería. - La actividad minera también es parte de la actividad económica de la población. El proyecto explorador Selene ya finalizó su tiempo de explotación, luego de 30 años. Actualmente, va a iniciar la explotación el proyecto minero Los Chancas que abarca los territorios de tres distritos. La actividad minera trae demanda de empleo temporal, en los diferentes proyectos que implementan, así como indirectamente la afluencia de gran cantidad de empleados de diferentes partes del país, exige a la población implementar restaurantes, hoteles, lavanderías, movilidad local, discotecas, bares entre otros servicios. Las autoridades de las comunidades de Tiaparo, Tapayrihua, Pocohuanca y Socco indican que "los jóvenes no quieren estudiar porque las mina les ha ofrecido trabajo, dicen que ahí le van a pagar billete y con eso van a poner su negocio". Por otro lado, los programas sociales que implementan las mineras fortalecen la infraestructura educativa, "Iscahuaca (distrito de Cotarusi) era un pueblo olvidado, ahora nuestro colegio tiene computadoras y también laboratorio para que aprendan nuestros alumnos, los maestros y los padres también, nos sentimos a gusto se han preocupado por la educación (refiriéndose a la compañía minera ARES)".

- Servicios de Salud.- Se prestan mediante 5 Centros de Salud y 33 Puestos de Salud distribuidos en todos los distritos, con infraestructuras en su mayoría inadecuadas e insuficientes. Los Centros de Salud cuentan con personal profesional calificado (médicos, obstetras, odontólogos, enfermeras y personal técnico), mientras los Puestos de Salud, que son la gran mayoría, están en la provincia, tienen personal a nivel de técnicos de enfermería, y pocos recursos. Esto implica una baja y deficiente atención con las consecuencias de fuertes grados de morbilidad y tasas significativas de mortalidad infantil y embarazo adolescente. Las campañas de promoción y concientización de los cuidados de la salud, así como la prevención de enfermedades y embarazos no deseados en los adolescentes (22\% solo en el año 2005) de la existencia de personal calificado para que asista y brinde información, charlas y capacitaciones a profesores, escolares, padres y la comunidad.

\section{Macrosistema}

Para nuestro estudio, se consideraron los siguientes factores (ver Gráfico 6).

4 Fuente Consolidado de PSMP Micro red Chalhuanca y Santa Rosa 2005. 
Gráfico 6. Organización estructural del modelo que conforma el Macrosistema.

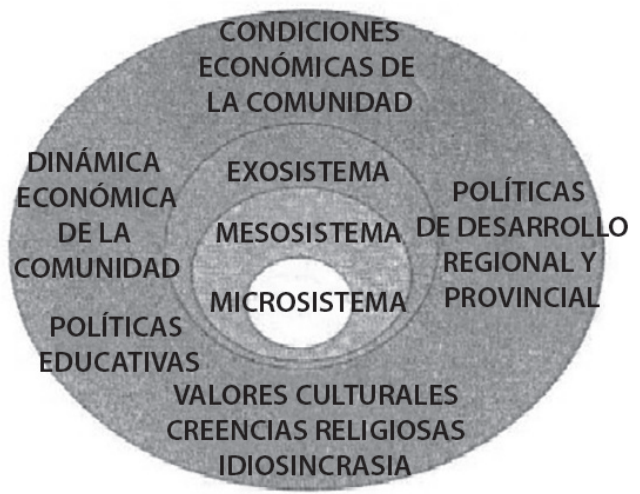

- Condiciones Socioeconómicas de la Comunidad.- El 70,8\% de la población Aymarina se encuentra en situaciones de pobreza y el 30,8\% en extrema pobreza (INEI, 2007). Durante la los años 1980, la población empieza a migrar a las zonas costeras, buscando lugares más seguros, ya que en aquella época Aymaraes era escenario de la guerra interna de los años 80 (informe de la Comisión de la verdad y reconciliación, 2003). Según el Censo Nacional de Población y Vivienda $2005^{5}$, el $35 \%$ de la población Aymaraes no cuenta con acceso a agua potable, el $62 \%$ no cuenta con servicios de desagüe y el $47 \%$ no cuenta con luz eléctrica. El nivel de desnutrición infantil en la zona, hasta el año 1999 era del $48 \%$ 6;7. El índice de desarrollo humano, para la provincia es de 0.5050, ubicado en el puesto 170 de 195 a nivel nacional (PNUD 2007). Los distritos más pobres de Aymares son beneficiarios de los programas estatales: Juntos, PRONAA, Vaso de Leche, PRONAMA, CRECER, SIS, etc.). La gran focalización de todos estos programas sociales en el ámbito de la provincia, indica claramente su condición económica.

Un gran porcentaje de la población no ha logrado ningún nivel de educación $(23,18 \%)$, asimismo el índice de analfabetismo de Aymaraes es de $24 \%$, en la población femenina alcanza el $38 \%^{8}$; Las personas con formación secundaria incompleta y completa representan el $16,86 \%$ y $9,3 \%$, respectivamente (INEI, 2005); una parte importante tiene una formación incompleta en nivel primario $(27,96 \%)$.

- Dinámica Económica de la Comunidad.- Las actividades económicas cotidianas en la zonas urbanas, principalmente en Chalhuanca, son el comercio, bodegas, locutorios, agencias de empresas de transporte, restaurantes, comercio ambulatorio, taxistas, mototaxistas, internet, bares, carpintería, molino de granos, talleres artesanales de tejidos, panaderías, venta de tuna

5 INEI 2005. Indicadores de la Educación Perú 2004. MINEDU 2005

6 INEI 2005

7 INEI 2005

8 INEI 2005 
y otros servicios que ofertan a las instituciones públicas o privadas (turismo, pensión, hospedaje, lavandería, entre otros). En la capital de la provincia y otros distritos que están al pie de la carretera interoceánica, cuentan hay demanda de estos servicios porque allí se encuentran las sedes o sucursales de las instituciones públicas y privadas (Municipalidad, Policía, Juzgado, Posta Médica, Fiscalía Centros Educativos entre otros). Económicamente, son estas zonas las más dinámicas. Los demás distritos rurales, basan su actividad económica en el trabajo de construcción en obras municipales, siendo estos temporales. La mayor parte del año depende de la venta de sus productos de temporada en ferias, local y provincial (maíz, papa, chirimoya, tuna, alfalfa, cuy, leche, tara, miel, lana de alpaca, textiles). Actualmente, existen programas o proyectos en ejecución, con financiamiento estatal o privado, que promueven el mejoramiento de la calidad de su producción ya sea agrícola, pecuaria o textil en cada uno de los distritos, y además su comercialización en mercados a nivel local (miel, queso, leche, yogurt, cuy, carne de alpaca), regional (miel, chirimoya) o nacional, (tara y papa nativa en los distritos de Sañayca y Soraya), respectivamente. La dinámica económica de la comunidad influye decisivamente en la inversión, capacidad de gasto y en la necesidad de empleo de las familias, y por ende en la deserción escolar.

- Políticas Educativas. - Las políticas educativas nacionales y/o regionales influyen decisivamente en los objetivos de la educación de cada región o provincia.

- Políticas de Desarrollo Regional y Provincial._ Las políticas de desarrollo regional de Apurímac consideran que Apurímac necesita una educación de calidad, moderna, con tecnología y adecuada infraestructura, pero además hace falta incrementar el presupuesto para la educación. Asimismo, la disminución de la desnutrición y la pobreza extrema son sus prioridades ${ }^{9}$. Lograr la organización de su pueblo bajo una identidad cultural es otra de sus prioridades. Estas políticas se convertirán en proyectos o perfiles de proyectos que, dependiendo de la capacidad de gestión administrativa del gobierno regional, logra tener expediente y aprobación para ejecutarse. Finalmente, su ejecución oportuna ${ }^{10}$, dependerá de la capacidad del ente ejecutor, de las exigencias del pueblo y de los vaivenes de la política de la región. Las políticas de desarrollo regional también influirán en las prioridades de lo que se enseñará a los estudiantes, si se contratará a profesores capacitados, conocedores del idioma a donde irán a servir, si dispondrán de capacitaciones o de alguna forma de que estos docentes no pierdan conexión con los programas de formación continua con que sí se benefician los profesores de zonas urbanas. Son las políticas también las que disponen si se destinará más o menos presupuesto para educación. Bajo estas políticas del gobierno central, los gobiernos regionales y las pequeñas unidades de gestión, conjuntamente con el gobierno local, harán su parte.

9 Minkay 2009

10 Minkay 2009 
- Valores, Cultura, Religión, Idiosincrasia.- La práctica de los valores del pueblo aymarino se ven expresadas en las relaciones denominadas minkayayni. La minka es una actividad que convoca a uno o más grupos solidarios con vínculos vecinales y familiares para brindar servicios de limpieza de acequias, trabajos de infraestructura local, actividad pública a la que generalmente se denominan "faenas". El ayni es una práctica que se da solo entre grupos familiares con la finalidad de desarrollar alguna actividad agrícola, festiva o doméstica. El pueblo sustenta su vida en la naturaleza, en lo que produce la tierra. Por ello expresa su cultura a través de las danzas, las mismas que interpretan las diversas formas de interacción con la naturaleza (siembra, cosecha, tiempos de lluvia, sequía) y también su forma sincrética de manifestar su fe, a través de la devoción a sus santos patrones. El 88\% de la población profesa el catolicismo sustentado en un amplio calendario festivo religioso (INEI, 2007). Todos los pueblos de Aymaraes celebran a un santo patrón y es motivo de una práctica común por esa región, el Yawarfiesta. Todas las fiestas están sustentadas por el ayni, desde meses atrás las familias recogen la leña en una celebración denominada llantakuscca, y son transportadas a la casa del alterado de ese año. El patrón de la provincia es el Señor de Ánimas. En su honor se celebra la denominada Fiesta Mayor de Apurímac en Chalhuanca, a finales de julio e inicios del mes de agosto con procesiones y tres tardes taurinas con toreros internacionales.

Los pueblos andinos de nuestro país, por mucho tiempo han sufrido la indolencia del gobierno y las autoridades que los han gobernado cercanamente. Hasta ahora sufren la postergación de su desarrollo y la desvalorización de su idioma y sus costumbres. El trabajo es para ellos una forma de educar en valores y responsabilidad a sus hijos. Contrariamente a lo que se divulga en las escuelas, que el trabajo es pernicioso para la educación de sus hijos. Esta situación no encuentra un punto de coincidencia, entre lo que tiene por cultura la comunidad y las expectativas de educación de sus hijos; y lo qué tiene por objetivo la educación básica regular en las escuelas, pensada-completamentepor gente ajena a sus necesidades y demandas.

\section{Diagrama Causal del Modelo Ecológico de Desarrollo Humano}

En esta dinámica se advierten las siguientes relaciones causales (ver Gráfico 7). 
Gráfico 7. Diagrama causal de la deserción en Aymaraes.

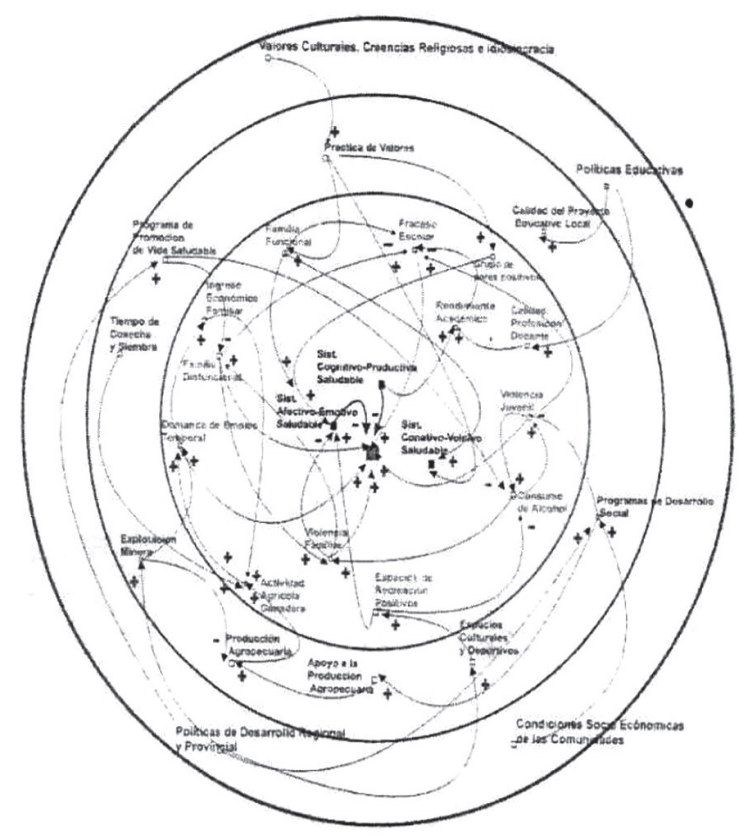

En el diagrama se muestran las relaciones causales de todas las variables. Cada una de ellas ubicado en un sistema ecológico. En el modelo dinámico se han establecido las relaciones causales entre las variables, considerando su nivel de importancia indicada como causal de la deserción escolar. La deserción escolar no está ubicada como una variable que interactúa en esta dinámica de los gráficos, más bien es una variable que emerge de la interacción de variables del sistema. Es un fenómeno social que emerge.

El sistema cognitivo-productivo saludable tiene una relación causal negativa con la deserción escolar. Un sistema cognitivo-productivo saludable depende de una relación causal positiva del rendimiento académico del estudiante, a su vez el rendimiento académico depende de las relaciones causales positivas de la calidad profesional docente, la cual depende positivamente de la calidad del Proyecto Educativo Local (regional y local), el cual a su vez va a depender de la relación positiva con las Políticas Educativas dictadas a nivel nacional.

Un sistema afectivo-emotivo saludable tiene una relación causal negativa con la deserción escolar. Este sistema depende de una relación causal positiva del tipo de familia con la que viva el estudiante (familia funcional), a su vez el tipo de familia dependerá de la práctica de valores propios de la comunidad. La violencia familiar tiene una relación causal negativa con el sistema efectivoemotivo saludable. Los espacios de recreación positivos en el entorno tiene una relación causal positiva con el sistema afectivo-emotivo saludable del estudiante, el cual depende de la relación causal positiva con los espacios 
culturales y deportivos existentes en el entorno, los que a su vez depende de las políticas de Desarrollo Regional y Provincial.

El sistema conativo-volitivo saludable del estudiante se relaciona negativamente con la deserción escolar. El sistema a su vez depende de una relación causal positiva de la Práctica de Valores, la que a su vez depende positivamente de los valores Culturales, creencias religiosas e Idiosincrasia de la población. El sistema conativo-volitivo se ve relaciona positivamente con la violencia juvenil, la que a su vez se relaciona negativamente con el fracaso escolar. Por otro lado, la violencia juvenil depende de una relación causal positiva del consumo de alcohol en la comunidad, a su vez tiene una relación positiva con la violencia Juvenil. Estas dos variables forman una relación causal de retroalimentación positiva dentro del sistema (bucle de retroalimentación positivo), son interdependientes una de otra. El consumo de alcohol tiene una relación causal negativa con la existencia de espacios de recreación positivos en la comunidad, la que a su vez va a dependen de la existencia de espacios culturales y deportivos considerados en políticas de desarrollo regional y provincial. El consumo de alcohol tiene una relación causal positiva con la Violencia Familiar, la cual influye en una relación causal negativa con el sistema de conativo-volitivo del estudiante.

La demanda temporal de empleo influye negativamente en el sistema conativo-volitivo saludable de los estudiantes. La demanda de empleo temporal depende de una relación causal positiva con la minería, actividad agropecuaria, y los tiempos de cosecha y siembra. Por otro lado, la actividad o producción agropecuaria depende negativamente de la minería (a mayor minería, menor actividad agrícola), la que dependerá positivamente de las Políticas de Desarrollo Regional y Provincial. La producción agropecuaria, a su vez, depende de los programas sociales implementados para tal fin, lo que va a depender positivamente de las condiciones socioeconómicas de la comunidad.

\section{DISCUSIÓN}

La deserción escolar es un fenómeno social que emerge de la interacción de muchas variables dentro de un sistema social determinado. De manera que los resultados de esta investigación muestran todas aquellas variables que, en interacción positiva o negativa, influyen en los sistemas que organizan la personalidad del estudiante. La organización saludable del sistema conativo-volitivo dependerá de la Práctica de Valores dentro la comunidad (Exosistema) y del tipo de familia en la que vive el niño (Mesosistema). La relación causal directa indica que un sistema conativovolitivo saludable se constituirá en una sociedad con práctica de valores positivos y viviendo en un hogar con una familia saludable. Alcázar y Valdivia (2005) indican que las cuestiones familiares y de salud (incluyendo el embarazo) son el segundo factor importante que determina la deserción. Como se ha descrito el sistema conativo-volitivo depende de la integración del comportamiento emotivo y 
el desempeño productivo en base a reglas morales que reflejen la estructura ética de la sociedad (Ortiz, 1994). Dependerá de la configuración de sus motivos, sus convicciones, sus expectativas, sus intereses, sus aspiraciones, sus prejuicios, sus responsabilidades y obligaciones, que el estudiante continúe estudiando satisfactoriamente o que deserte. La vida del estudiante dentro de una familia disfuncional (con bajo nivel socioeconómico, conflictiva, que no cumple con su función de protección y cuidado a sus hijos) organizará una pobre valoración respecto a sus intereses y expectativas sobre la educación, la cual es sensible a los conflictos que se le presentan. Para el caso de las estudiantes mujeres, salir embarazada significará dejar de estudiar para dedicarse a trabajar o realizar las tareas propias de la maternidad. Para el caso de los varones, un fracaso escolar significará desertar la escuela para dedicarse a otra actividad, dentro o lejos de la comunidad. Los valores culturales del macrosistema no afectan directamente sobre los motivos, expectativas o decisiones del estudiante, pero tienen una relación causal positiva, porque estos se expresan en la vida de familia y es donde el estudiante interioriza estos valores y lo práctica en la sociedad. La violencia juvenil es una variable que expresa la configuración disfuncional en el entorno familiar. Esta se relaciona positivamente con el consumo de alcohol en la comunidad. El consumo de alcohol, que es una práctica generalizada en las actividades agrícola-ganaderas, tiene sus efectos en el brote y la continuación de la violencia juvenil. Esta relación constituye lo que en dinámica de sistemas se denomina bucles de retroalimentación positivos, la cual significa que las dos variables tienden a aumentar en forma exponencial en interdependencia (Aracil, 1995). La positiva relación causal entre el consumo de alcohol y la violencia familiar, va a configurar negativamente las motivaciones, expectativas y decisiones del estudiante, propiciando la deserción escolar. Es una variable de largo alcance, que no influye directamente, sino que forma parte del continuum del desarrollo de esa persona.

El sistema cognitivo-productivo Tiene que ver con la forma de representar su ambiente, desplazarse y operar en él, va a depender de los conocimientos, de los niveles perceptivos, y atencionales del estudiante (Ortiz, 1994). Tal es así que el rendimiento académico del estudiante va a depender de una relación causal positiva con las posibilidades pedagógicas del docente, la infraestructura del centro educativo y los objetivos del colegio. Las posibilidades pedagógicas del docente permitirán o no, lograr una enseñanza integrando los saberes previos de los estudiantes, respetando su cultura, e influirá positivamente en el buen desempeño académico del estudiante, y en sus expectativas de continuar estudios superiores. Las posibilidades pedagógicas del docente van a depender positivamente de las disposiciones de las políticas dadas a nivel nacional, regional y/o local. Los incentivos a los profesores, la capacitación permanente y el reconocimiento a su práctica pedagógica, influirá en mejorar su práctica profesional.

Las disposiciones afectivas van a organizar el sistema afectivo-emotivo del estudiante (Ortiz, 1994). La organización de los sentimientos personales, interpersonales y extra personales de los estudiantes, influirá en su forma de responder ante situaciones de los diferentes entornos del mesosistema. Así, el estado de 
ánimo, su nivel de tolerancia a la frustración, las actitudes con que haga frente a sus aprendizajes, las dificultades en la escuela, la percepción y expectativa de los profesores hacia su desempeño académico, la relación con sus pares, la percepción de su familia y de su comunidad, la configuración saludable de este sistema tiene una relación negativa con la deserción escolar, es decir mientras el alumno logre una adecuada configuración de este sistema de su personalidad, el riesgo de desertar la escuela será menor. En cambio, existen factores como la violencia familiar, el tipo de familia disfuncional que influencian negativamente en el desajuste de este sistema. Los estudios realizados por Alcázar y Valdivia (2005) indican que los problemas familiares son una causa muy importante de la deserción escolar. Una familia con constantes prácticas de violencia de parte de los padres hacia los hijos o violencia entre los padres, va a dificultar una adecuada configuración de los aspectos emotivos y afectivos de los hijos. Asimismo, una familia que funciona desajustadamente, exigirá de los hijos cumplir roles que no son compatibles con las exigencias de la escuela. Cuidado de los hermanos menores, hacerse cargo de las labores domésticas, buscar trabajo para mantener a la familia o cuidar de los sembríos o los animales. En este tipo de familias, las más desfavorecidas son las niñas mujeres, a quienes muchas veces se les retira de la escuela o el colegio para que cumpla las labores domésticas que hacen falta. El tipo de familia y la violencia familiar dependen a su vez de relaciones positivas con la práctica de valores propios de la comunidad; así una comunidad con altos índices de alcoholismo propiciará el aumento de la violencia familiar y el tipo de familias disfuncionales.

Los espacios culturales y deportivos existentes en el entorno influencian positivamente en una saludable configuración del sistema afectivo-emotivo del estudiante. Estos espacios de recreación facilitan la interacción del estudiante con sus pares y el aprendizaje de patrones conductuales positivos en un ambiente de expresión cultural; el cual beneficiaría el sistema cognitivo-productivo del estudiante, mejorando sus motivaciones y expectativas respecto a su educación actual y la continuidad de la misma. La existencia de estos espacios culturales y deportivos va a depender de las políticas de desarrollo, tanto regional, provincial o local.

\section{OTROS ASPECTOS DE LA DISCUSIÓN}

Este trabajo representa un esfuerzo pionero aplicando tres perspectivas confluyentes:

La teoría ecológica del desarrollo humano, la teoría de la personalidad de Pedro Ortiz Cabanillas y el análisis de dinámica de sistemas. Este enfoque pretende superar las limitaciones del enfoque de causa - efecto, por un enfoque que permita analizar la complejidad de los factores en juego en el análisis de un fenómeno considerándolo parte de un sistema, estableciendo sus interacciones y reestructuraciones, aplicándolo entonces al fenómeno de la deserción escolar como parte de un sistema dinámico. Es importante el uso tanto de diagramas causales como principalmente el de diseño de bucles de realimentación positivos que conservan 
y refuerzan la verticalidad; como negativos que van a ser contrarios al desarrollo del mismo fenómeno. Sin embargo, la utilización de este enfoque hace necesario algunas reflexiones.

En primer lugar, el análisis de un sistema implica su consideración como una unidad funcional, cuyos elementos al variar implican la variación de elementos contenidos en los otros subsistemas concéntricos. Esta apertura permite ver las relaciones desde los macrosistemas pasando por los meso y micro, para ver justamente su incidencia en el sistema de la personalidad y en las decisiones del sujeto. En el sistema del análisis funcional, se presenta el fenómeno estructurándose aquí y ahora, sin superar el análisis de factores. Creemos que es un análisis valioso pero que adolece en sí de no encontrar categorías que permitan estructurar las relaciones que se dan dentro, desde los sistemas más amplios hasta los microsistemas donde se expresa en las relaciones de interacción, es decir en las relaciones intersubjetivas.

$\mathrm{Al}$ considerar la persona, como si fuera cualquier otro organismo que "va adentrándose progresivamente y reestructurando el medio en el que vive", y "tiene una concepción cambiante del ambiente ecológico, en su capacidad de descubrir, mantener o modificar las propiedades del ambiente en que se encuentra"; se utiliza una concepción reduccionista; se olvida que el carácter específico de la actividad humana, es su carácter de práctica, es decir, actividad realizada por un sujeto socializado que actuará y interactuará en cada nivel como sujeto que se transforma y se auto transforma, expresando en cada momento de su actuación parte de las relaciones sociales que estructuran la realidad, desde los exosistemas pasando por los meso y microsistemas; expresándose en cada persona concreta relaciones sociales con especificaciones de sexo, cultura y clase, estructuradas todas ellas principalmente por las relaciones de clase.

Por cuanto la práctica, es decir la forma de actividad propia del ser humano, se desarrolla fundamentalmente en dos ámbitos: uno fundante, el trabajo que estructura el carácter de las relaciones sociales, y sus formas específicas como concreción de un determinado modo de organizar la producción y obviamente el consumo. Y la otra forma de la práctica en que los sujetos participan es el mantenimiento, reestructuración y transformación de las relaciones sociales; es decir su participación, activada o bloqueada en el manejo de la organización de las condiciones de su existencia. Y por último la mediación de estas formas de práctica y las categorías elaboradas (ideológicas) a nivel del Macrosistema para dar cuenta de esa práctica, que pueden o no expresar el carácter objetivo y real de esta para los sujetos que la realizan. Sin esas tres categorías estructurantes es difícil sistematizar el carácter de las relaciones que se establecen entre el niño alumno desertor y los diversos niveles de análisis de la realidad, por lo que en un futuro análisis deben ser incorporados. Doise (1982), en su planteamiento afín al nuestro, consideraba que las investigaciones experimentales de los procesos psicosociales enfrentaban una seria limitación derivada de lo que hemos señalado. El vaciar de contenido las categorías es uno de los problemas de utilizar categorías de tipo reduccionista. 
Otro aspecto de carácter metodológico a nivel del sistema que organiza los factores que inciden en la deserción escolar, tiene que ver con analizar necesariamente las contradicciones entre dos mundos existentes en la realidad de Aymaraes. Plantear solamente la distribución de la población como urbano-rural no nos permite comprender el carácter de las contradicciones que se vienen dando en Aymaraes como en el resto del país. El tránsito en el Perú de una población predominantemente rural a una población predominantemente urbana, definida claramente ya a partir de la década del noventa, tiene que ver con la extensión de las relaciones capitalistas de producción. El mantenimiento de las relaciones de una economía natural, casi de autoconsumo en Aymaraes, es una contradicción evidente y se expresa en el predominio de la población rural. Es necesario señalar que el desarrollo de las relaciones capitalistas, ha sido posible, solo por el importante barrimiento de las relaciones semifeudales producto de la guerra interna como culminación de otros procesos de larga data; y los procesos económicos impulsados por la política neoliberal de la globalización. Esto implica la contradicción entre las necesidades de superación de esa incipiente economía capitalista, que se expresa en la capital de Aymaraes, con una propuesta que permita impulsar el desarrollo de la región sin la cual no tiene sentido una educación escolar. La no solución de esto que es un indicador decisivo de las limitaciones de este sistema. No debemos olvidar que la escuela como reivindicación popular y como necesidad del sistema, es consustancial al desarrollo de una sociedad industrial capaz de integrar la mano de obra en forma masiva, y donde ello no ocurre, la población no puede abandonar sus medios tradicionales de producción sin una perspectiva de ocupación segura, ahí se da que la alfabetización y la escuela aparecen superfluas.

\section{AGRADECIMIENTOS}

Expresamos nuestro profundo agradecimiento al pueblo de Aymaraes y sus instituciones educativas, que nos acogieron durante dos años y nos permitieron recoger la información para este trabajo, a través de entrevistas y talleres. Nuestra decisión de realizar esta investigación en Aymaraes, nació de las constantes motivaciones y apoyo moral del profesor Leoncio Solórzano Acuña, quién además fue el artífice para lograr nuestra estancia en aquella provincia, mediante un convenio específico entre la Municipalidad Provincial de Aymares y la Facultad de Psicología de la Universidad Nacional Mayor de San Marcos. También agradecemos al Ing. Celedonio Méndez, profesor del Curso de Dinámica de Sistemas de la Universidad Nacional de Ingeniería, quien nos permitió aprender sobre la metodología de la investigación, aceptándonos como alumnos libres en sus clases en esa universidad. Finalmente, queremos agradecer a Enver Oruro Puma por su apoyo constante, desde el inicio de este trabajo. A él le debemos las valoraciones cualitativas para la construcción del modelo y la revisión del mismo. 


\section{REFERENCIAS BIBLIOGRÁFICAS}

Alcázar L. (2008). Asistencia y deserción en escuelas secundarias rurales del Perú. (pp 41-81). En: Contribuciones empiricas para el debate. Martín Benavides, ed. Grupo de Análisis para el Desarrollo Lima - Perú disponible www.

Ames P. y Uccelli F. (2008). Formando futuros maestros: observando las aulas de institutos superiores pedagógicos públicos (pp. 131-176). (Ibidem) www.

Ames, P (2008). Construyendo equidad y democracia en la escuela: evaluación de dos modelos de intervención educativa en Lima y Cusco (pp. 365-402) (Ibidem) www.

Aracil, J (1995). Dinámica de Sistemas. Madrid: Ingeniería de Sistemas .

Bronferbrenner U (1979). Toward and Experimental Ecology of development, pp. 513-531. American Psychology. Jul.

Espinoza P.G, Oruro E.p, Andrade E Aguilar M (2010). La Neurociencia social base de microsistema para el modelo Dinámico Ecológico del Desarrollo Humano en zonas altoandinas del Perú. Hontanares. Vol. XII N. ${ }^{\circ} 1$, pp. 5367. Enero-diciembre.

Espíndola E y León (2002). La deserción escolar en América latina: Un tema prioritario para la agenda regional. Revista iberoamericana de educación N. ${ }^{\circ} 30$, pp. 36-62.

Forrester J (2000). Comportamiento contratuitivo de los sistemas sociales. Traducido por grupo de Dinámicas de Sistemas de ITESM, Monterrey México primera edición del año 1971.

Lavado P. y Gallegos J (2005). La dinámica de la deserción escolar en el Perú: un enfoque usando modelos de duración: Lima, Universidad del Pacífico.

Ortiz Cabanillas Pedro (1994). El sistema de la personalidad. Orión, Lima. 